\title{
Two-channel Kondo Lattice Model on a ladder studied by the Density Matrix Renormalization Group Method
}

\author{
Juana Moreno ${ }^{1}$, Shaojin $\mathrm{Qin}^{2}$, P. Coleman ${ }^{3}$, and $\mathrm{Lu} \mathrm{Yu}{ }^{4,2}$ \\ ${ }^{1}$ Dept of Physics \& Astronomy, Northwestern Univ., 2145 Sheridan Rd, Evanston IL 60208, USA \\ 2 Institute of Theoretical Physics, P. O. Box 2735, Beijing 100080, People's Republic of China \\ 3 Serin Laboratory, Rutgers University, 136 Frelinghausen Road, Piscataway, NJ 08854-8019, USA. \\ ${ }^{4}$ International Center for Theoretical Physics, P.O. Box 586, 34100 Trieste, Italy
}

(October 26, 2018)

\begin{abstract}
Using the density matrix renormalization group (DMRG) method we study a two-channel Kondo lattice model on a half filled ladder. Our model involves an on-site s-wave and a nearest neighbor dwave coupling between the local moments and the conduction electrons on the ladder. By changing the relative strength of the two Kondo interactions we examine the evolution of the system from a conventional Kondo insulator with a singlet at each site to a new kind of semimetallic state formed by overlapping of Zhang-Rice-like singlets. The DMRG is used to study how the spin and charge correlation functions evolve between these two regimes.
\end{abstract}

Pacs numbers:75.30.Mb,71.30.+h,71.20.Rv,71.27.+a

\section{INTRODUCTION}

The interaction between localized spins and delocalized conduction electrons plays a central role in the physics of heavy fermion materials, 1 Kondo insulators 13 and many other highly correlated electron systems. The Kondo lattice model (KLM) is probably the simplest theoretical model that describes this physics.

Considerable insight into local moment materials has been obtained by studying the one-dimensional Kondo lattice. 6 Although some properties are intrinsic topne dimensional models, such as the nesting instabilities $\bar{E}$, other aspects such as the insulating behavior at half filling and the Fermi surface expansion by the local moment $\$$ are also inherent to three-dimensional systems. Recently, the theoretical study of one-dimensional models has also proved a most useful tool in the analysis of an increasing number of low-dimensional materials. The comparison of experimental findings with exact numerical results helps to evaluate the adequacy of basic models and to quantify the relevance of additional effects such as the importance of the orbital electronic degrees of freedom.

The earliest claims of quasi-one-dimensional behavior in Kondo lattife intermetallic compounds date back to the seventies, 10 but only recently, with the synthesis of the quasi-one-dimensional molecular conductor $(D M E T)_{2} \mathrm{FeBr}_{4}$, has a clear-cut experimental realization of a 1D Kondo lattice been found 11 This compound displays features of a strongly correlated electron system. It shows a metal-insulator transition at $200 \mathrm{~K}$, which appears to be driven by the interaction between the $\pi$ conduction electrons of the DMET donor sheets and the localized $\mathrm{Fe}^{3+}$ spins. 12

A class of heavy fermion systems, known as Kondo insulaters, display insulating properties at low temperatures.3 One of the interesting aspects of a Kondo lattice is the emergence of insulating ground- states. The ground state of the conventional Kondo lattice model is a non-magnetic insulator at half-filling, with a gap in both spin and charge excitation spectrum.t In this way, the half-filled Kondo lattice provides a simple model for the Kondo insulator, where the coherent Kondo effect forms a highly renormalized band-insulator. However, the smallest gap Kondo insulators, CeNiSn and $C e R h S b$, do not fit into this simple scheme: they appear to develop gapless excitations.13 The narrow-gap Kondo insulators can be regarded as an example of a half-filled Kondo system with a gapless ground state due to the presence of interactions that are absent in the conventional Kondo lattice model. 14.15

A simple model which displays a metallic ground state at half-filling is the "d-wave Kondo model". This is a variant of the conventional Kondo model where each local spin couples through the exchange interaction with a d-wave orbital formed by the nearest-neighbor electrons. This interaction favors the formation of Zhang-Rice-like singlet 16 between each spin and the nearest conduction electron orbitals. The superposition of these extended singlets may establish a new kind of semimetallic state, which may be relevant to explaining the metallic phase of $(D M E T)_{2} \mathrm{FeBr}_{4}$. Another interesting feature of the dwave Kondo model is the introduction of spin-dependent hopping processes, where an electron is able to jump to the next-nearest-neighbor site by flipping the spin of the local moment. This kind of interaction plays a role in other organic compounds such as the magnetic graphite intercalation compounds.17

Motivated by the need to understand how a metalinsulator transition arises from pure exchange interactions between the itinerant and localized electrons, this paper examines a Kondo model with two spin-exchange channels sharing a single conduction band: an on-site s-wave (Fig. if) and a nearest neighbor d-wave coupling (Fig. 2). Electron-electron interactions, such as Hund's interactions, can induce the opening of new spin- 
exchange channels between the local moments and the conduction electrons. 20 For an impurity magnetic ion, the Kondo effect develops exclusively in the strongest screening channel due to the local symmetry which preserves the channel quantum number of scattered electrons. In a lattice, however, a conduction electron can change symmetry channels as it moves from one spin site to another. Therefore, the development of a Kondo effect in one channel no longer excludes the possibility of a Kondo effect developing coherently in other channels.

Our goal is to determine whether there is a range of exchange coupling for which such a two-channel Kondo model displays gapless excitations at half-filling. If this is the case, the competition between the $\mathrm{d}$ - and s-wave Kondo couplings might induce new intermediate phases between the d-wave Kondo ground state (Zhang-Rice singlets at each site) and the conventional Kondo ground state (s-wave Kondo singlets). It has been argued that even a composite superconducting phase could appear in the KLM if the local moments interact with a single conduction band via two orthogonal scattering channels with the same spatial parity.21

In highly correlated electron systems, it is essential to go beyond the mean-field level to understand their low-energy properties. We apply the density matrix renormalization-group (DMRG) method22 to calculate the energy spectrum and ground state correlations of this two-channel KLM. This real-space technique has proved to be remarkably accurate for one-dimensipnal systems such as the Kondo and Anderson lattices.23 25 To check the character and energy range of the elementary excitations we have also performed a variational calculation in the d-wave limit.

Section II introduces the Hamiltonian and the parameters of our model, the scaling law used to analyze the results and the numerical technicalities of the calculation. We present our results for the ground state energy and the spin and charge excitation gaps in section III. In Section IV, we discuss the spin and charge correlations of the ground state and some characteristics of the excited states. Section V is dedicated to a comparison between our results and a variational calculation and finally, section VI presents our conclusions.

\section{MODEL}

The minimal one dimensional model with two Kondo exchange channels of different symmetry and the same parity is a ladder model. The exchange interactions between the local moments and the electrons are an on-site s-wave (Fig. 11) and a nearest neighbor d-wave coupling (Fig. 2). Unlike other multichannel Kondo models, our model involves a single conduction electron band and Kondo singlets can form for any value of the couplings. Electrons and local moments are located at sites $\vec{R}_{i}^{k}=i \hat{x}+k \hat{y}$, where $\mathrm{k}=0,1$. We impose open bound- ary conditions in the $x$ direction. Along the $y$ axis, the system can be considered a periodic lattice with a period of two lattice spacings. The Hamiltonian is:

$$
H=H_{0}+H_{s}+H_{d}
$$

where $H_{0}$ is the electronic kinetic energy given by,

$$
H_{0}=-t \sum_{i, k}\left(\psi_{i+1 \alpha}^{k \dagger} \psi_{i \alpha}^{k}+\psi_{i \alpha}^{k+1^{\dagger}} \psi_{i \alpha}^{k}+\text { h.c }\right),
$$

where $t$ denotes the hopping matrix element, and the operator $\psi_{i \alpha}^{k^{\dagger}}$ creates a conduction electron on site $\vec{R}_{i}^{k}$ with spin $\alpha . H_{s}$ is the conventional s-wave Kondo interaction:

$$
H_{s}=J_{s} \sum_{i, k}\left\{\vec{S}_{i}^{k} \cdot \psi_{i \alpha}^{k^{\dagger}} \frac{\vec{\sigma}_{\alpha \beta}}{2} \psi_{i \beta}^{k}\right\} \quad(\mathrm{s}-\text { channel })
$$

where $J_{s}$ is the strength of the antiferromagnetic s-wave coupling, $\vec{S}_{i}^{k}$ and $\vec{s}_{i}^{k}=\psi_{i \alpha}^{k^{\dagger}}\left(\vec{\sigma}_{\alpha \beta} / 2\right) \psi_{i \beta}^{k}$ denote the spin of the local moment and the conduction electron at position $\vec{R}_{i}^{k}$, respectively.

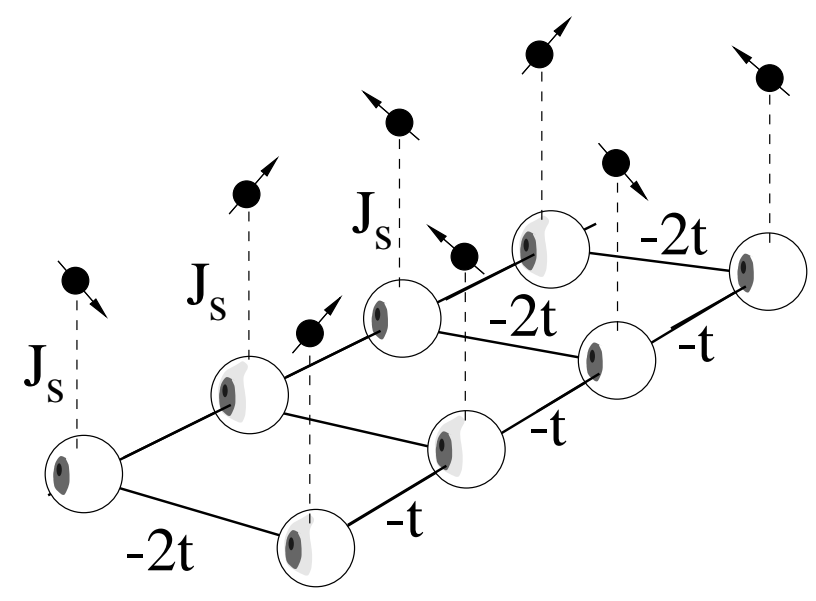

FIG. 1. Ladder illustrating the electronic hopping and the s-wave Kondo interaction between each local spin (arrows) and the conduction electron at the same site (spheres).

The last term, $H_{d}$, represents the d-wave Kondo channel:

$$
\begin{array}{r}
H_{d}=J_{d} \sum_{i, k}\left\{\vec{S}_{i}^{k} \cdot d_{i \alpha}^{k \dagger} \frac{\vec{\sigma}_{\alpha \beta}}{2} d_{i \beta}^{k}\right\} \quad(\mathrm{d}-\text { channel }), \\
\text { where } \quad \mathrm{d}_{\mathrm{i} \alpha}^{\mathrm{k} \dagger}=\frac{\left(\psi_{\mathrm{i}+1 \alpha}^{\mathrm{k}^{\dagger}}+\psi_{\mathrm{i}-1 \alpha}^{\mathrm{k}^{\dagger}}-\gamma \psi_{\mathrm{i} \alpha}^{(\mathrm{k}+1)^{\dagger}}\right)}{\sqrt{2+\gamma^{2}}} .
\end{array}
$$

The operator $d_{i \alpha}^{k \dagger}$ creates an electron with "d-wave symmetry" in the " $\gamma$ " Wannier state at site $\vec{R}_{i}^{k}$. For $\gamma=1$ this state has pure d-wave symmetry but since the onedimensional ladder breaks the $90^{0}$ rotation symmetry of the lattice, we explore a more general class of states by allowing $\gamma$ to become a free parameter in the model (in this 
way our "d-channel" involves an admixture of d and extended s-wave character). Note that the d-wave spin exchange involves spin-dependent hopping processes where the electron hops between next-near-neighbor sites via the exchange of spin with the local moments.

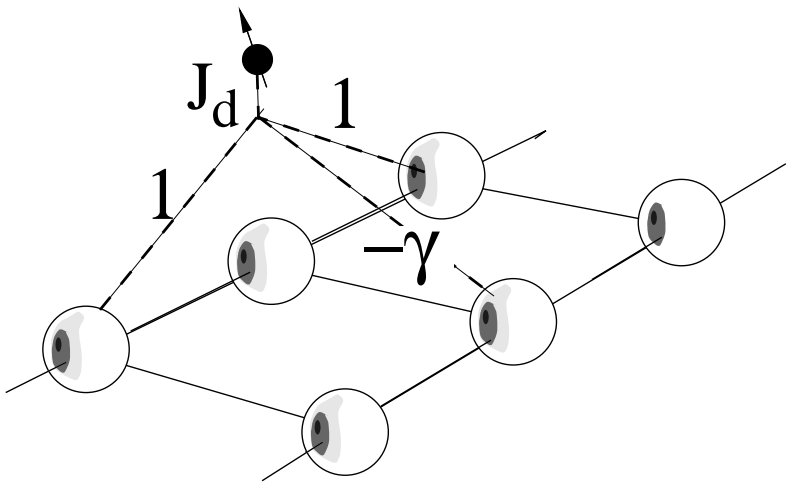

FIG. 2. Ladder illustrating the d-wave Kondo interaction between each local spin and the three near-neighboring electrons. Note that the coupling is $J_{d}$ along the chains and $-\gamma J_{d}$ along the rungs of the ladder.

Our interest lies in the half-filled case where the total number of conduction electrons is equal to the number of lattice sites. Finite size studies on our model are facilitated by using the strong-coupling limit to reduce the feasibility of antiferromagnetic instabilities, although, as we discuss later, we can not completely eliminate them. Thus, we use the following values of the parameters, $t=0.01$ and $|J|=\sqrt{J_{s}^{2}+J_{d}^{2}}=1$. A finite hopping $t$ is required to ensure the smooth distribution of the electronic density. We have studied three values of the transverse exchange parameter, $\gamma=2,1,0.5$, and several values of the ratio between the $\mathrm{d}$ - and s-wave exchange couplings, $J_{d} / J_{s}$.

We use the finite-chain DMRG algorithm22 up to ladders of 12 rungs, using up to 100 optimized states for each block. In contrast to the infinite system method, the finite system algorithm gives more accurate results, but an asymptotic extrapolation to the infinite system is necessary. We use the following scaling law,26 which is appropriate for a massive mode,

$$
\Delta(L)=\sqrt{\Delta^{2}(L=\infty)+v^{2} \frac{\pi^{2}}{(L+1)^{2}}}
$$

where $\Delta(L)$ is the gap for a system of length $L$. This allows us to define a correlation length, $\xi=v / \Delta(L=\infty)$, for the spin and charge modes of the system. In the DMRG calculations, the truncation of the Hilbert space might lead to deviations from the above asymptotic behavior 27 The deviations can be substantial for small gaps, especially when the dimension of the Hilbert space used in the computation is not sufficiently large.28

In our computation, the largest truncation errors are of the order of $10^{-7}$ for the conventional s-wave limit, while for the biggest $J_{d}$ values considered, the errors are as big as $10^{-3}$. As the ratio $J_{d} / J_{s}$ increases, the truncation error becomes larger since the Kondo screening cloud extends across substantially longer distances and the magnetic fluctuations grow. As a result, the accuracy of our calculation is significantly reduced in this limit.

Ground state energy

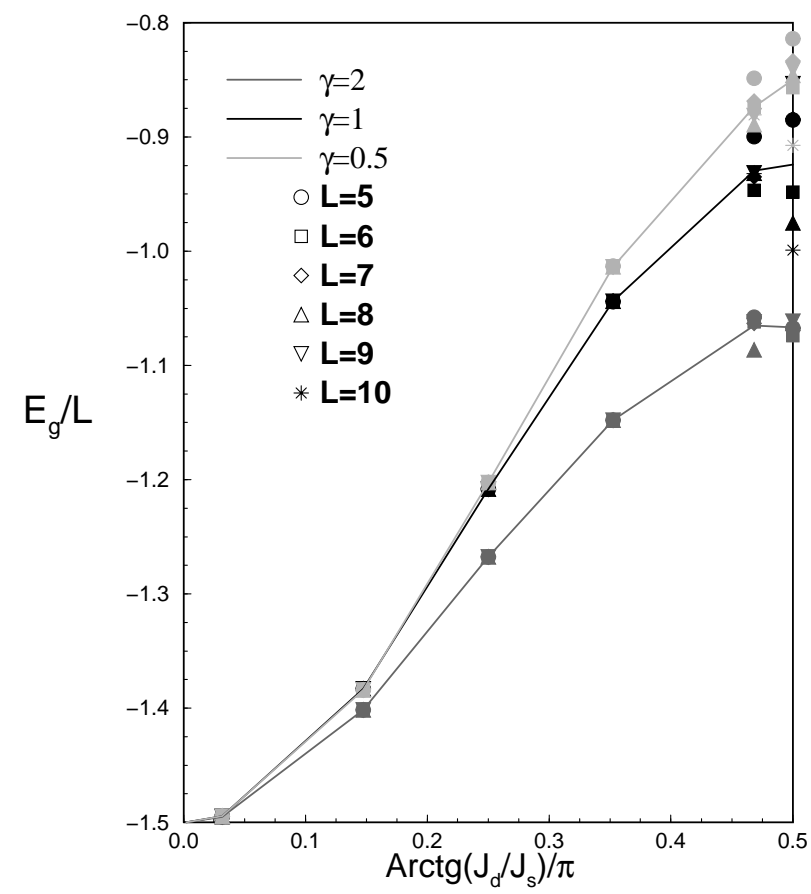

FIG. 3. Ground state energy per rung of the two-channel Kondo ladder model versus $\arctan \left(J_{d} / J_{s}\right) / \pi$ for transverse exchange parameter $\gamma=2$ (dark gray symbols), $\gamma=1$ (black symbols) and $\gamma=0.5$ (light gray symbols). We have plotted the results for ladders of 5 (circles), 6 (squares), 7 (diamonds), 8 (up-triangles), 9 (down-triangles) and 10 (stars) sizes. Note that the lines in this plot are only guides to the eyes.

\section{GROUND STATE ENERGY AND SPIN AND CHARGE EXCITATION GAPS}

Let us define the ground-state energy in a given spin-S subspace for a finite ladder with L-rungs by $E_{g}\left(L, N_{c}, S\right)$, where $N_{c}$ is the number of conduction electrons. Given that we are interested in the insulating ground state, we will consider a half-filled band with one electron per site and a total of $N_{c}=2 L$ electrons. The spin gap is defined as the energy difference between the ground state and the lowest spin excitation that changes the total spin quantum number by one: $\Delta_{s}(L)=E_{g}(L, 2 L, S=$ 1) $-E_{g}(L, 2 L, S=0)$.

Similarly, the charge gap is the energy difference between the ground state and the lowest pure charge excitation, which changes the total carrier number by two and keeps the spin quantum numbers fixed, $\Delta_{c}(L)=$ $E_{g}(L, 2 L+2,0)-E_{g}(L, 2 L, 0)$. 
Fig. 3 displays the energy of the ground state as a function of $\arctan \left(J_{d} / J_{s}\right) / \pi$ for the three values of $\gamma$ explored in our calculation. Results for several lattice sizes have been plotted. The ground state energy increases with the strength of the d-wave coupling for any value of $\gamma$ and reaches the highest values when $\gamma=0.5$.

Let us now discuss the results for the ground state and excited state energies of the conventional Kondo lattice model, $J_{s}=1$ and $J_{d}=0$. In the strong coupling limit, the energies can be calculated by using a perturbative expansion in $t / J$. To second order in this small parameter, the ground state energy per rung is $E_{g}=-(3 / 2) J-\left(4 t^{2} / J\right)=-1.5004$, the spin gap is $\Delta_{s}=J-\left(20 t^{2} / J\right)=0.998$, and the charge gap is $\Delta_{c}=(3 / 2) J-4 t+\left(9 t^{2} / J\right)=1.4609$, where we have used the parameters of our model. Our numerical results are: $E_{g}=-1.50040, \Delta_{s}=0.9981$ and $\Delta_{c}=1.4614$, where we have used the scaling discussed in the previous section. Given the almost complete agreement between the perturbation expansion and our numerical results, the accuracy of our computation in this limit is confirmed.

In the d-wave limit, $J_{s}=0, J_{d}=1$, the ground state energies for the three different values of $\gamma$ are the following:

$$
\begin{aligned}
& -\gamma=2, E_{\text {ground }}^{\text {rung }} \sim-1.05 \\
& \text { - } \gamma=1, E_{\text {ground }}^{\text {rung }} \sim-0.93 \\
& \text { - } \gamma=0.5, E_{\text {ground }}^{\text {rung }} \sim-0.88
\end{aligned}
$$

These are reasonable values for the ground state energy in the d-wave strong coupling limit. For large $\gamma$, the system will tend to form singlets along the rungs of the ladder. The energy of such configuration is: $E_{\text {ground }}^{\text {rung }}=$ $2 \frac{\gamma^{2}}{2+\gamma^{2}}(-3 / 4)$. For $\gamma=2$, the ground state energy is just $E_{\text {ground }}^{\text {rung }}=-1$. The value obtained in our calculation is rather close to this simple limit. In the opposite case of $\gamma=0$, let $t=0$, there are four decoupled chains. The spins at each chain tend to form spin-singlets with their two neighboring electrons with exchange coupling $J_{d} / 2=1 / 2$. Therefore, the ground state energy per rung is related with the ground state energy of four Heisenberg chains: $E_{\text {ground }}^{\text {rung }}=4(1 / 2)(1 / 4-\ln 2)=-0.886$. The value obtained for $\gamma=0.5$ in our calculation is again close to this simple limit. The numerical ground state energy for $\gamma=1$ is between the upper bound of -1 and the lower bound of -0.886 . Therefore, our result agrees with this simple physical argument.

Figures 4, 5 and 6 display the charge and spin gaps versus $\arctan \left(J_{d} / J_{s}\right) / \pi$ for $\gamma=2,1$ and 0.5 , respectively. Results for several lattice sizes have been plotted. The gap resulting from using the scaling law (6) is also shown. In addition, the first column of tables II and II displays the spin and charge correlation lengths, respectively, derived from the scaling parameters of equation (6), $\xi=v / \Delta(L=\infty)$.

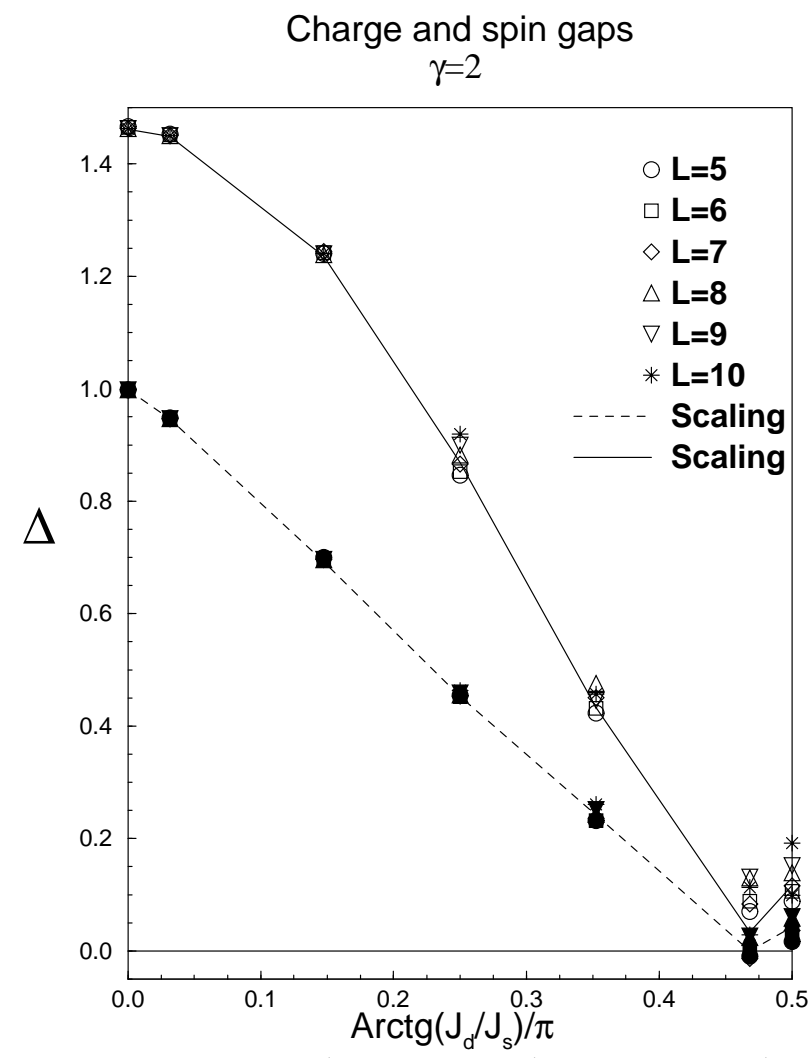

FIG. 4. Charge gap (white symbols) and spin gap (black symbols) versus $\arctan \left(J_{d} / J_{s}\right) / \pi$ for a d-wave transverse exchange parameter $\gamma=2$. We have plotted the results for ladders of 5 (circles), 6 (squares), 7 (diamonds), 8 (up-triangles), 9 (down-triangles) and 10 (stars) sizes. Also, the charge (solid line) and spin (dashed line) gaps obtained using the scaling procedure are shown.

The behavior of the spin and charge gaps for any value of the parameter $\gamma$ is similar. By increasing the d-wave coupling, the system evolves to a state with much smaller spin and charge gaps for any $\gamma=2,1,0.5$. Comparing figures 4,5 and 6, it can also be seen that $\Delta_{s}$ and $\Delta_{c}$ in the $\mathrm{d}$-wave limit increase as $\gamma$ decreases. So, a transverse exchange parameter of $\gamma=2$ is the most favorable value to develop a gapless region in the phase space. In fact, the smallest values of the gaps correspond to $\gamma=2$ and $J_{d} / J_{s}=10$, where by using scaling (6) we are able to infer values of $\Delta_{\text {spin }}=2 \cdot 10^{-7}$ and $\Delta_{\text {charge }}=0.034$. Our belief is that an increase in values of the d-wave Kondo exchange coupling, which implies a delocalization of the singlet formed between the spin and the conduction electrons, induces a reduction of the energy gaps. We think that for $\gamma<1$, the ladder sustains a spin and charge gap. However, for $\gamma>1$, the system develops gapless excitations at a finite value of the ratio $J_{d} / J_{s}$. Unfortunately, the accuracy of the calculation is not enough to get precise results in the $J_{d} / J_{s} \rightarrow \infty$ limit. We can not conclusively establish our expectation that a gapless region is present for any value of the ratio $J_{d} / J_{s} \gtrsim 10$ when $\gamma>1$. Also, we do not know whether spin and charge gaps drop to zero precisely at the same ratio of 
$J_{d} / J_{s}$ or the spin excitation becomes gapless before the charge gap closes.

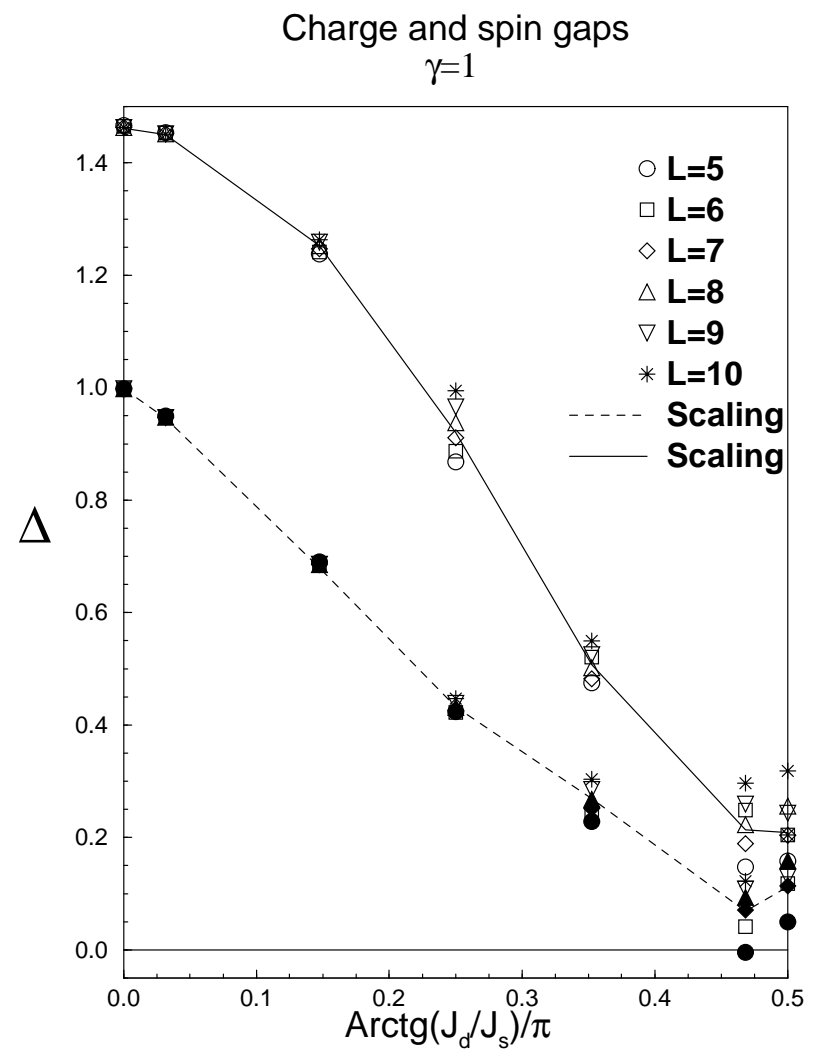

FIG. 5. Charge gap (white symbols) and spin gap (black symbols) versus $\arctan \left(J_{d} / J_{s}\right) / \pi$ for a transverse exchange parameter $\gamma=1$. Notations are the same as before.

Other models also display gapless excitations at halffilling, such as the attractive Kondo-Hubbard model, where, in addition to the Kondo exchange, the conduction electrons have an on-site attraction.29 In the strong coupling limit of that model, there is a range of parameters where both excitation gaps become equal and then drop to zero. However, in our model, although both excitation gaps are strongly reduced, the ratio between them grows when $J_{d} / J_{s}$ increases. This fact implies that the time scale of the collective spin fluctuations that destroy the antiferromagnetic long-range order increases faster with $J_{d} / J_{s}$ than the characteristic time scale of the conduction-electron propagation.

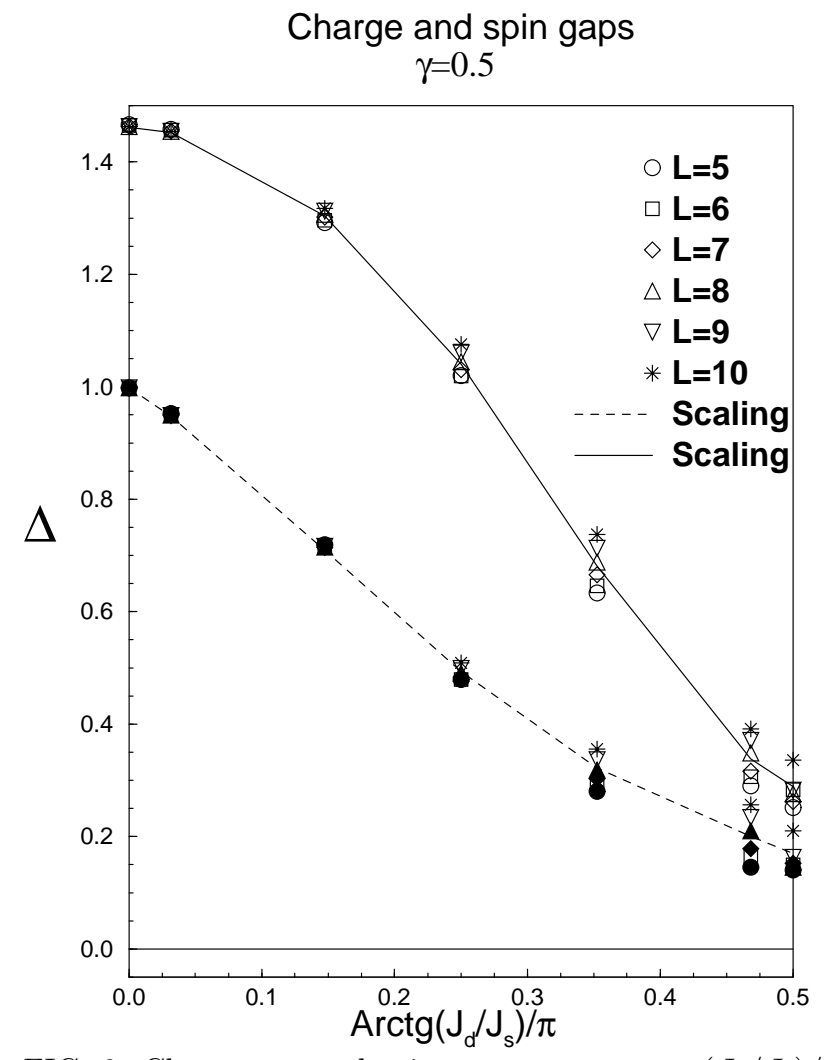

FIG. 6. Charge gap and spin gap versus $\arctan \left(J_{d} / J_{s}\right) / \pi$ for a transverse exchange parameter $\gamma=0.5$. Notations are the same as before.

Let us conclude this Section commenting on the numerical difficulties of the problem. The computation is much more involved in the d-wave limit $\left(J_{s}=0, J_{d}=1\right)$. The gap is, at least, two orders of magnitude smaller. Also, the correlation length in the ground state increases roughly by two orders of magnitude, and longer ladders are required to reach the convergent regime with respect to the chain length. The number of degrees of freedom in the Kondo lattice model is eight states per site. Therefore, the exact diagonalization can be carried out only for rather short ladders (five rungs in our case).

In addition, as it is the case for the one dimensional swave Kondo lattice 23 we find that the Ruderman-KittelKasuya-Yosida (RKKY) interaction increases in importance as $J_{s}$ decreases. For small $J_{s}$, large $J_{d}$, the system is so close to an instability that the numerical inaccuracy associated with larger ladder sizes is able to induce spurious antiferromagnetic ordering of the lattice, because the states with and without long range order are very close in energy.

Also, in the d-wave limit, the smallness of the hopping term makes difficult the redistribution of the local densities and, as consequence, the determination of the smooth and energetically lowest electronic states. Our approach to improve the numerical accuracy has been to make several self-consistent sweepings for each block to guarantee the smooth redistribution of the local densities. 


\section{SPIN AND CHARGE CORRELATIONS IN THE GROUND STATE. CHARACTERISTICS OF THE SPIN EXCITED STATES}

The DMRG method enables us to calculate the equaltime spin and charge correlation functions. We minimize the effects of the open boundary by evaluating correlations between the central site and other sites along the ladder. To clarify the nature of the correlations, we consider the pure d-wave exchange model with no hopping $(t=0)$. In this case, the ladder decouples in two disconnected zig-zag chains. If only d-wave exchange is present, the spin sitting at position " 0 " in Fig. 7 correlates only with the electrons at the black sites, not with the ones in the gray chain. Correlations between the central spin and electrons at the gray sites are induced by either swave exchange or hopping terms. We use black and gray colors to display the different correlation functions. For example, the spin-spin correlation $\left\langle\vec{S}_{0}^{0} \cdot \vec{s}_{i}^{k}\right\rangle$ between the central spin and the electron at site $\vec{R}_{i}^{k}$ will be display in black if $\vec{R}_{i}^{k}$ belongs to the black chain and in gray in the opposite case.

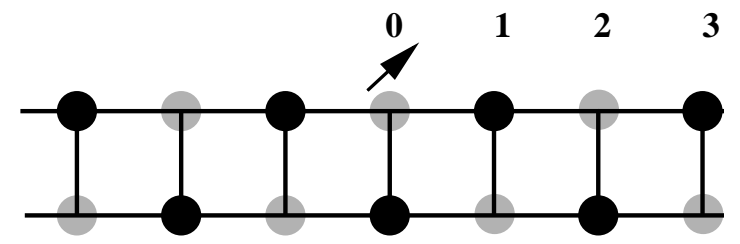

FIG. 7. The black sites couple with the spin through the d-wave Kondo channel. The gray sites couple through the conventional s-wave channel.

In the calculation of the correlation functions, we have used the local spin symmetry of the ground state $\left(\left\langle\rho_{i \uparrow}\right\rangle=\left\langle\rho_{i \downarrow}\right\rangle\right)$ and the fact that the ladder is half-filled. 30 We only discuss results for a d-wave transverse exchange value of $\gamma=2$, but the correlations for the other values of $\gamma$ exhibit similar characteristics.

Figure 8 shows the spin-spin correlation between the local moment at the center of the ladder and electrons at different sites, $\left\langle\vec{S}_{0} \cdot \vec{s}_{i}\right\rangle$, as a function of the electron position for several values of the ratio $J_{d} / J_{s}$. It can be seen how the ground state evolves with increasing values of $J_{d} / J_{s}$ from a conventional insulating state with strong on-site correlations to a new state with large spinspin correlation along the rungs and longer correlation lengths.

In the s-wave limit (top-left graph in Fig. 8) the value of the on-site correlation is as large as $\left\langle S_{0}^{z 0} s_{0}^{z 0}\right\rangle=$ -0.2499 . The correlations with the nearest neighbors in this case are extremely small, $\left\langle S_{0}^{z_{0}^{0}} s_{1}^{z^{0}}\right\rangle \sim\left\langle S_{0}^{z 0} s_{0}^{z 1}\right\rangle \leq$ 0.001 . This confirms the localized character of the ground state when only the on-site exchange is present. As $J_{d} / J_{s}$ grows, the correlation with the electrons at black sites gradually increases, and the correlation with the gray sites decreases. When only d-wave exchange is present $\left(J_{s}=0, J_{d}=1\right)$ the correlation with the electrons in the black chain becomes dominant. The correlation with the electron along the rung reaches its highest value of $\left\langle S_{0}^{z 0} s_{0}^{z 1}\right\rangle=-0.16$.

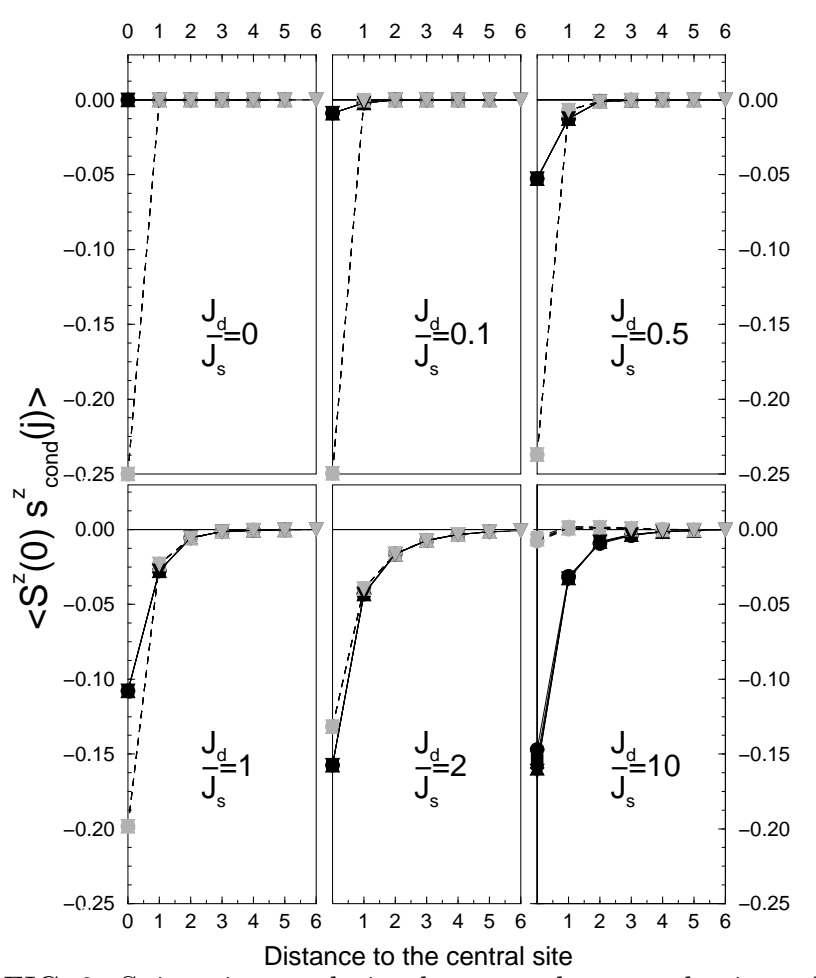

FIG. 8. Spin-spin correlation between the central spin and the electron at rung $j$ as a function of the distance $j$. Correlations with electrons at gray (black) sites are shown in gray (black). The transverse exchange is $\gamma=2$. Values for several ladder sizes are represented with different symbols as in previous figures.

We are also able to infer typical values of the spin correlation lengths by fitting the spin-spin correlation functions. An exponential fit, $\left\langle\vec{S}_{0} \cdot \vec{s}_{j}\right\rangle=C \exp \left[-j / \xi^{\text {spin }}\right]$, is appropriate for most of the ratios $J_{d} / J_{s}$. However, for $J_{d} / J_{s} \gtrsim 10$ a power law fit, $\left\langle\vec{S}_{0} \cdot \vec{s}_{j}\right\rangle=C /\left[\left(j / \xi^{\text {spin }}\right)^{\nu}+1\right]$, more accurately describes the correlations along the gray chain, as it can be seen in the top graph of Figure (9). The transition from an exponential to a power law decay suggests the development of gapless spin excitations in the system, in agreement with our previous conclusions. Table fisplays the correlation lengths between the central moment and the electrons at the gray (black) zigzag chain, $\xi_{\text {gray }}^{\text {spin }}\left(\xi_{\text {black }}^{\text {spin }}\right)$, for different values of the ratio $J_{d} / J_{s}$. The correlation length derived from the scaling of the excitation gap $\left(\xi^{\text {sin }}=v / \Delta\right)$ is also shown. We notice that $\xi^{\text {spin }}$ and $\xi_{\text {gray }}^{\text {spin }}$ have similar values and both increase with the ratio $J_{d} / J_{s}$. On the other hand, $\xi_{\text {black }}^{\text {spin }}$ is roughly constant for all the values studied. 

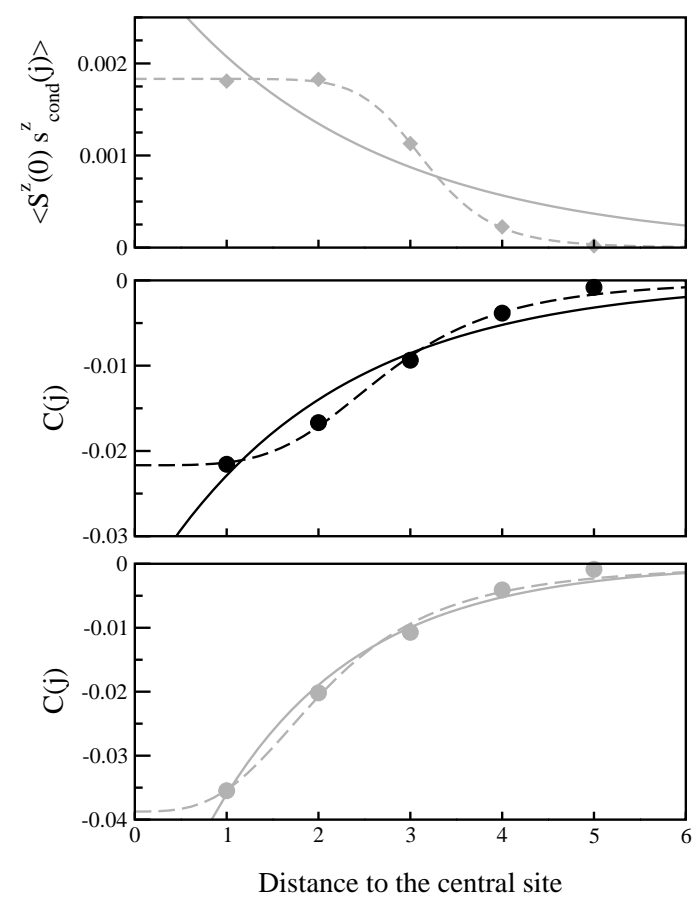

FIG. 9. Comparison between exponential and power law fits to the DMRG results for the correlation as function of the distance to the central site for $J_{d} / J_{s}=10$ and a ladder of 10 sites. Top graph, middle graph and bottom graph display the numerical data (circles), exponential (solid line) and power law (dashed line) fits for the spin correlation along the gray chain, the charge correlation along the black chain and the charge correlation along the gray chain, respectively

TABLE I. Spin-spin correlation lengths for different values of the ratio $J_{d} / J_{s}$ (first column). The second column displays the lengths derived from the scaling law. The third and fourth columns show the correlation lengths between the central local moment and electrons at the gray and black chains, respectively.

\begin{tabular}{|c|c|c|c|}
\hline$J_{d} / J_{s}$ & $\xi^{\text {spin }}=v / \Delta$ & $\xi_{\text {gray }}^{\text {spin }}$ & $\xi_{\text {black }}^{\text {spin }}$ \\
\hline 0 & 0.02 & 0.061 & 0.67 \\
\hline 0.1 & 0.13 & 0.14 & 0.66 \\
\hline 0.5 & 0.33 & 0.281 & 0.66 \\
\hline 1 & 0.48 & 0.47 & 0.72 \\
\hline 2 & 0.71 & 0.88 & 0.81 \\
\hline 10 & - & 3.17 & 0.64 \\
\hline
\end{tabular}

Let us now discuss the characteristics of the charge correlation functions. Figure 10 displays the charge correlation, $C(j)=\langle\rho(0) \rho(j)\rangle-\langle\rho(0)\rangle\langle\rho(j)\rangle$, as function of $j$, the position of the second electron. The chargecharge correlation is practically zero everywhere for a pure s-wave exchange. This behavior agrees with the strong coupling limit of the Kondo model. The introduction of a finite d-wave coupling induces an on-site charge correlation that becomes quite big in the d-wave limit, $C(0) \sim 0.24$. The correlation with the remaining sites in the ladder is smaller and negative. This large onsite charge-charge correlation is related to the non-local character of the singlet state in the d-wave channel, as we discuss in the next Section.

We can also notice in Fig. 10 that the correlation length for both zig-zag chains grows with $J_{d} / J_{s}$. Table III shows the correlation lengths between the electron at the central site and electrons in the gray (black) zig-zag chain, $\xi_{\text {gray }}^{\text {charge }}\left(\xi_{\text {black }}^{\text {charge }}\right)$, for different values of the ratio $J_{d} / J_{s}$. Also, the correlation length derived from the scaling $\left(\xi^{\text {charge }}=v / \Delta\right)$ is shown. All the correlation lengths grow with the ratio $J_{d} / J_{s}$, but their precise values are different. As in the spin case, the charge correlation lengths have been obtained using an exponential fit in the region $J_{d} / J_{s}<10$ and a power fit for $J_{d} / J_{s} \geq 10$. Middle and bottom graphs in Figure (9) show a comparison between exponential and power law fits for $J_{d} / J_{s}=10$.

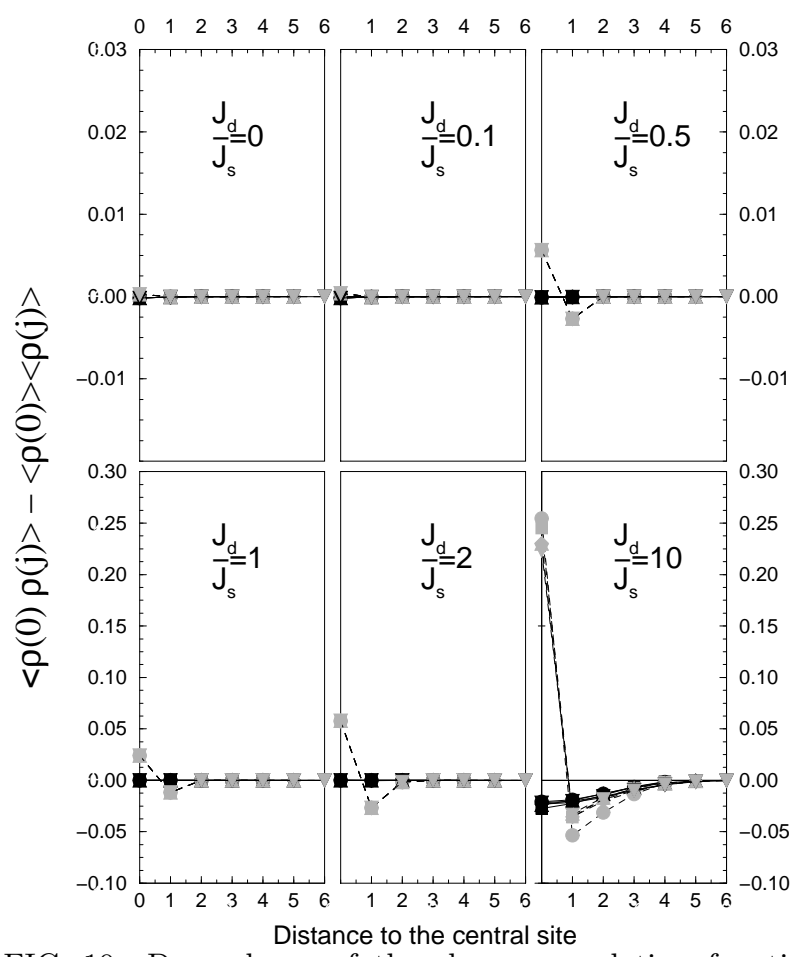

FIG. 10. Dependence of the charge correlation function on the distance to the central site for a transverse exchange $\gamma=2$. Correlations with electrons at gray (black) sites are shown in gray (black). Note the factor of ten difference in the vertical scale between the top and bottom graphs. Values for several ladder sizes are presented with different symbols as in previous figures.

From the analysis of the correlation functions, we conclude that there is a phase transition in the two-channel Kondo lattice when the transverse exchange parameter is larger than unity. Spin and charge correlation functions evolve from the ones typical of a conventional Kondo lattice to those of a d-wave Kondo ladder with gapless 
spin and charge modes. The value of the spin correlation length derived from the scaling law (eq. (6) ) correlates only with the spin correlation length along the gray chain. The spin correlation length along the black chain appears to be determined by the hopping interaction rather than by the d-wave exchange coupling. On the other hand, the charge correlation lengths of the two zig-zag chains (black and gray) behave similarly and are in agreement with the lengths associated with the charge gap scaling. In any case, we have to keep in mind that results for larger ratios of $J_{d} / J_{s}$ are likely to be less reliable given that for these values $2 \xi \sim L_{\text {diag }}$, where $L_{\text {diag }}=5$ is the length for which an exact diagonalization is carried out.

TABLE II. Charge-charge correlation lengths for different values of the ratio $J_{d} / J_{s}$. The second column displays the lengths derived from the scaling law. The third and fourth columns show the correlation lengths between the central site and sites in the gray and black chains, respectively.

\begin{tabular}{||c|c|c|c||}
\hline \hline$J_{d} / J_{s}$ & $\xi^{\text {charge }}=v / \Delta$ & $\xi_{\text {gray }}^{\text {charge }}$ & $\xi_{\text {black }}^{\text {charge }}$ \\
\hline 0 & 0.146 & 0.43 & 0.67 \\
0.1 & 0.150 & 0.10 & 0.70 \\
0.5 & 0.25 & 0.17 & 0.95 \\
1 & 0.34 & 0.24 & 1.77 \\
2 & 0.81 & 0.36 & 1.32 \\
10 & 8.56 & 2.09 & 2.75 \\
\hline \hline
\end{tabular}

Let us conclude by discussing some characteristics of the spin excited states. Fig. 11 displays the value of the third component of the total spin in each rung as a function of the distance between the rung and the center of the ladder for $J_{d} / J_{s}=0$, open circles, and $J_{d} / J_{s}=10$, open diamonds. In the insets, the z-component of the spin of the local moments, $\left\langle S_{j}^{z}\right\rangle$, and the conduction electrons, $\left\langle s_{j}^{z}\right\rangle$, are displayed. The results correspond to a ladder of 12 sites with open boundary conditions.

In the case of the conventional s-wave limit, the spin of the local moments, $\left\langle S_{j}^{z}\right\rangle$, and of the electrons, $\left\langle s_{j}^{z}\right\rangle$, are almost identical for any site in the ladder, as it can be seen in the top inset of Fig. 11. The magnitude of the spin is also equally distributed between the lower and upper chains. The local and electronic spins followa square sine function, appropriate for a massive mode,26

$$
\begin{aligned}
\left\langle S_{j, \text { total }}^{z}\right\rangle= & \left\langle S_{j}^{z}+s_{j}^{z}\right\rangle \sim 2\left\langle S_{j}^{z}\right\rangle \sim 2\left\langle s_{j}^{z}\right\rangle \sim \\
& \frac{1}{L_{\text {open }}}\left[\sin \frac{\pi\left(j+\left(L_{\text {open }} / 2\right)\right)}{L_{\text {open }}}\right]^{2}
\end{aligned}
$$

where $j$ is the position under consideration and $L_{\text {open }}$ is the length over which the spin excitation extends, which is longer than the ladder length. By making a twoparameter fit, $\left\langle S_{j, \text { total }}^{z}\right\rangle=C\left[\sin \frac{\pi\left(j+\left(L_{\text {open }} / 2\right)\right)}{L_{\text {open }}}\right]^{2}$ we infer $L_{\text {open }}=12.16$ and a normalization coefficient $\mathrm{C}=0.0822$, in agreement with the value of $1 / L_{\text {open }}$. This agreement can also be clearly seen in Fig. 11, where the solid line is the fitting function.

In contrast with the case of the conventional Kondo ladder, the local and the conduction spins display very different behavior in the d-wave limit (see the bottom inset in Fig. 11). The third component of the local spin of each rung is always positive; it decreases from the center $\left(\left\langle S_{0}^{z}\right\rangle \sim 0.44\right)$ to the end site of the ladder $\left(\left\langle S_{L}^{z}\right\rangle \sim 0.16\right)$. On the other hand, the magnitude of the z-component of the electronic spin is always negative and ranges between -0.28 and -0.14 . However, the sum of both spin densities, $\left\langle S_{j, \text { total }}^{z}\right\rangle$ behaves similarly to the case of only on-site exchange coupling, although it is clear that the dispersion of the data is larger (Fig. 11). By using the same twoparameter fitting, $\left\langle S_{j, \text { total }}^{z}\right\rangle=C\left[\sin \frac{\pi\left(j+\left(L_{\text {open }} / 2\right)\right)}{L_{\text {open }}}\right]^{2}$, we obtain a length of $L_{\text {open }}=13.02$ and a normalization coefficient $\mathrm{C}=0.0766$, which should be compared with $1 / L_{\text {open }}=0.0768$.

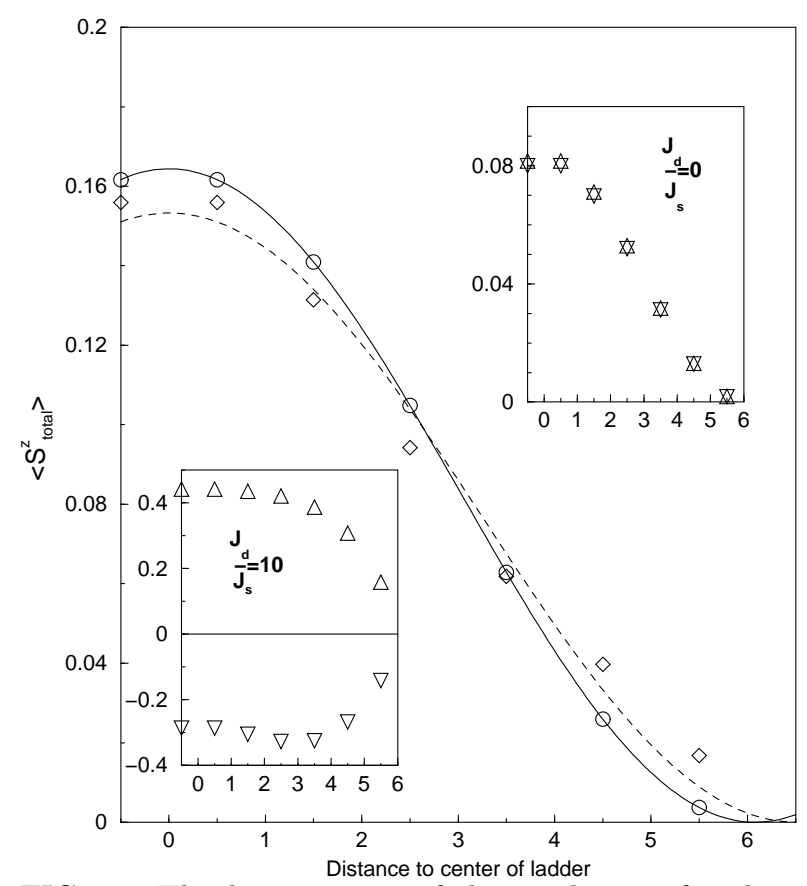

FIG. 11. Third component of the total spin of each rung as a function of the distance to the center of the ladder for $J_{d} / J_{s}=0$, open circles, and $J_{d} / J_{s}=10$, open diamonds. The lines represent the best fit to the expression: $S_{\text {total }}^{z}=a_{0}\left(\sin \left[\pi\left(j+\left(L_{\text {open }} / 2\right)\right) / L_{\text {open }}\right]\right)^{2}$. The solid line fits the $J_{d} / J_{s}=0$ points, dashed line fits the $J_{d} / J_{s}=10$ set of points. The insets display the z-component of the spin of the local moments (triangles up) and the conduction electrons (triangles down) for these two values of the ratio between the exchange couplings.

\section{VARIATIONAL CALCULATION}

In order to gain more insight into the nature of the ground state in the d-wave limit, we have performed a variational calculation. Variational wave functions have 
been successfully used in the conventional Kondo lattice model.31. We restrict ourselves to a simpler Hamiltonian with only $\mathrm{d}$-wave coupling. In this case, as we have discussed previously, the ladder decoupled in two disconnected chains (Fig. 7). We can label the sites of one of the zig-zag chains just by their $j$ coordinate. So, the Hamiltonian for one of the decoupled chains is reduced to:

$$
H_{d}=\frac{1}{2\left(2+\gamma^{2}\right)} \sum_{j} \vec{S}_{j} \cdot\left(\mathbf{d}_{j, s}^{\dagger}(-\gamma) \vec{\sigma}_{s s^{\prime}} \mathbf{d}_{j, s^{\prime}}(-\gamma)\right)
$$

with $\mathbf{d}_{j, s}^{\dagger}(x)=\psi_{j+1, s}^{\dagger}+\psi_{j-1, s}^{\dagger}+x \psi_{j, s}^{\dagger}$, where $\psi_{j, s}^{\dagger}$ creates a conduction electron with spin $s$ on site $j$.

Our variational wave function is the product of a Zhang-Rice-like singlet at each site:

$$
\left|\Psi_{0}\right\rangle=\prod_{l}\left(|\uparrow\rangle_{l} \mathbf{d}_{l, \downarrow}^{\dagger}(\alpha)-|\downarrow\rangle_{l} \mathbf{d}_{l, \uparrow}^{\dagger}(\alpha)\right)|0\rangle,
$$

where $|\uparrow\rangle_{l}\left(|\downarrow\rangle_{l}\right)$ indicates that the lth spin is in the state with $S_{z}=+1 / 2\left(S_{z}=-1 / 2\right)$. The operator $\mathbf{d}_{l, \sigma}^{\dagger}(\alpha)$ creates a non-orthogonal Wannier state which overlaps with the states at neighboring sites. We allow $\alpha$ to vary over values that differ from $\gamma$.

By decomposing $\alpha$ into its magnitude and its phase, $\alpha=|\alpha| e^{i \phi}$, we find that the energy minimum for any transverse exchange parameter $(\gamma)$ and length of the chain is always reached when $\phi=\pi$. Table [III displays the values of $|\alpha|$ for which the variational energy becomes minimum and the value of that minimum. For $\gamma=2$, the asymptotic minimum is in reasonable agreement with the computed value of -1.05 . The variational energy obtained for $\gamma=0.5$ is also remarkably close to the value obtained in the DMRG calculation $\left(E_{\text {ground }}^{\text {rung }} \sim-0.88\right)$. However, the variational energy obtained for $\gamma=1$ is too high; it is even higher than the energy of the configuration when half of the spins form singlets with one of their nearest neighbors $\left(E_{\text {ground }}^{\text {rung }}=-3 / 4\right)$. The energy minimum for $\gamma=2$ is a very shallow one, and there is a large region of $|\alpha|$ values where the energy is very close to -1 . In contrast, for $\gamma=1$ and 0.5 the energy minimum is reached at finite values of the parameter $|\alpha|$, and a scaling in $1 / L$ is necessary to obtain the ground state energy of an infinite system.

TABLE III. Results of our variational calculation for the studied transverse exchange parameters. The second and third columns show the magnitude of $|\alpha|$ where the energy

\begin{tabular}{|c|c|c|}
\hline$\gamma$ & $|\alpha|$ & $E_{\text {ground }}^{\text {rung }}$ \\
\hline 2 & $\infty$ & -1 \\
\hline 1 & 0.45 & -0.71 \\
\hline 0.5 & 0.26 & -0.89 \\
\hline
\end{tabular}
minimum is achieved and its value, respectively.

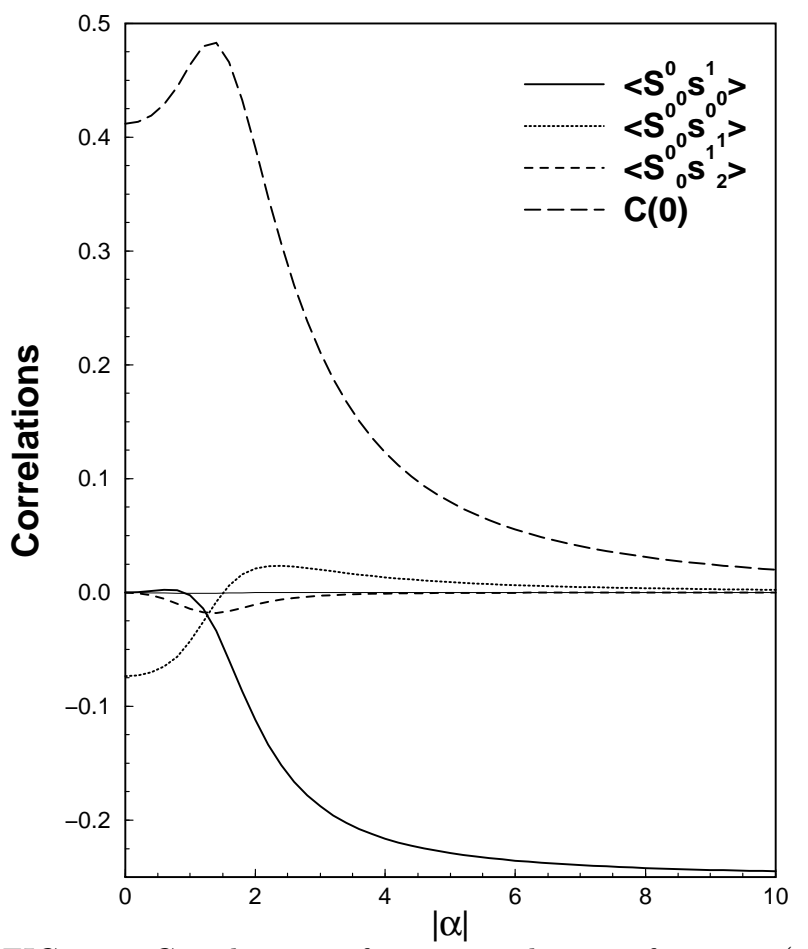

FIG. 12. Correlations of variational wave function (9) versus the parameter $|\alpha|$. Spin correlation between the central moment and the electron along the rung $\left(\left\langle S_{0}^{z}{ }_{0}{ }^{z}{ }_{0}^{1}\right\rangle\right)$, solid line; correlation with the nearest electron along the chain $\left(\left\langle S_{0}^{z 0} s_{1}^{z 0}\right\rangle\right)$, dotted line, and correlation with the next-next-nearest electron along the rung $\left(\left\langle S_{0}^{z}{ }_{0}^{0} s_{2}^{z}\right\rangle\right)$, dashed line. The on-site charge-charge correlation, $C(0)=\langle\rho(0) \rho(0)\rangle-\langle\rho(0)\rangle\langle\rho(0)\rangle$, is also displayed (long-dashed line).

We have also calculated the charge-charge and spinspin correlations as functions of $|\alpha|$, for $\phi=\pi$. The spin-spin correlation between the local spin at the center of the ladder and the two nearest, and next-next-nearest electrons sitting in the black chain are displayed in Fig. 12, together with the on-site charge-charge correlation at the central spot. Since gray and black chains are totally decoupled in our model (Hamiltonian (8)), correlations with electrons at the gray sites are absent. From this plot we conclude that extended Kondo models, where the moments couple with electrons on other (rather than it's own) sites, display extended spin correlations and strong on-site charge-charge correlations. There is a large range of values of the variational parameter $|\alpha|$ where the spin and charge correlations reach big values. For example, for $|\alpha|=2.8, C(0)=0.24$ and $\left\langle S_{0}^{z 0} s_{0}^{z 1}\right\rangle=-0.18$. These values are in good agreement with those found in our DMRG calculation for $\gamma=2$. So, it is reasonable to think that the ground state of the d-wave Kondo ladder, at least for some values of the transverse coupling, can be understood in terms of extended singlets on each lattice site (Eq. 9). 


\section{CONCLUSIONS}

Motivated by experimental1 and theoretical considerations about the phase diagram of Kondo lattice models 21 we have msed the density matrix renormalization group method 22 to study the strong coupling limit of a Kondo lattice model with two different interactions: the conventional on-site Kondo exchange $\left(J_{s}\right)$ and a d-wave exchange coupling $\left(J_{d}\right)$. Our purpose has been to map the phase diagram of this model and sort out whether or not it displays a phase transition.

By increasing the ratio between the two couplings $J_{d} / J_{s}$, the system smoothly evolves from a conventional Kondo insulator ground state, where a local singlet is formed at each site, to a ground state with much smaller spin and charge excitation gaps. For small values of $\gamma$ less than unity, our results suggest that the system preserves a spin and charge gap for all values of the $J_{d} / J_{s}$ ratio. However, when the parameter $\gamma$ is increased to values larger than unity, there is clear evidence to suggest that the system develops gapless excitations for $J_{d} / J_{s} \sim 10$, suggesting a phase transition. For a transverse exchange parameter of $\gamma=2$ and within the accuracy of the calculations, both the spin and charge gap become zero at this point. However, numerical difficulties related to the proximity to an antiferromagnetic instability preclude us from resolving definitively whether these gaps are zero for $J_{d}>10 J_{s}$ corresponding to a phase transition into a metallic phase, or whether the gaps re-establish small but finite values at $J_{d}>10 J_{s}$, corresponding to a critical point at $J_{d} / J_{s}=10$. Our intention is to increase the Hilbert space and the length of the ladder to be able to get more accurate values of the excitations gaps in this limit.

This semimetallic ground state with gapless spin and charge excitations can be understood as a superposition of the extended singlets formed between each local moment and its three nearest-neighbor electrons. The characteristics of the ground state excitations change with $J_{d} / J_{s}$. In particular, we find that as $J_{d} / J_{s}$ rises, the ratio of charge to spin gap $\Delta_{c} / \Delta_{s}$ is enhanced. Simultaneously, the system develops strong spin-spin correlations between the local moment and the electron along the rung and large on-site charge-charge correlations. The typical correlation lengths increase with the $J_{d} / J_{s}$ ratio. Also the transition from an exponential to a power law decay in the spin and charge correlations for $\gamma=2$ and $J_{d} / J_{s}>10$ supports the existence of a gapless phase.

Additional insight into the results at large $J_{d}$ has been obtained from a variational calculation. By writing down a variational wavefunction in which an extended singlet forms between each moment and the three neighboring electrons in the ladder, we obtain variational ground state energies that are close to our DMRG values for $\gamma=2$ and 0.5 . Our variational ansatz also displays large spin-spin correlations along the rungs and strong on-site charge-charge correlations as found in the DMRG results.
It would be interesting in future work to examine the effect of three-leg and four-leg ladders on the above results. By increasing the number of legs, the full pointgroup symmetry of the two-dimensional limit is gradually restored, permiting d-wave singlets to delocalize without admixing into extended s-wave pairs. This may permit the gapless phase to be reached at lower values of the ratio $J_{d} / J_{s}$. Indeed, the vanishing spin and charge gaps observed in our ladder model may become a full-fledged phase transition in truly two-dimensional systems. 21

We are grateful for the support provided by the Abdus Salam International Center for Theoretical Physics (Trieste, Italy), where this work was initiated and all numerical calculations were carried out. We thank Natan Andrei, Daniel Cabra, Andreas Honecker, Andres Jerez, Karine Le Hur, Edmond Orignac and Alexei Tsvelik for valuable discussions. We thank John Cooper for mentioning to us the earlier work of J.B. Dunlop (Ref. 9 10) and Victor Yakovenko for pointing out Ref. 11. This research was partially supported by NSF grants DMR 9705473, DMR 9972087 and DMR 91-20000 through the Science and Technology Center for Superconductivity. S. Qin was partially supported by the Chinese NSF. P. Coleman is supported by NSF grant DMR 9983150.

${ }^{1}$ For a review of heavy fermion materials, see e.g. N. Grewe and F. Steglich, Handbook on the Physics and Chemistry of Rare Earths, vol. 14, (1991), R. H. Heffner and M. R. Norman, Comments Cond. Mat. Phys. 17, 361 (1996)

${ }^{2}$ P. S. Riseborough, Adv. Phys. 49, 257 (2000)

${ }^{3}$ G. Aeppli and Z. Fisk, Comments Cond. Mat. Phys. 16, 155 (1992)

${ }^{4}$ H. Tsunetsugu, M. Sigrist and K. Ueda, Rev. Mod. Phys. 69, 809 (1997)

${ }^{5}$ See, for example Organic superconductors by T. Ishiguro, K. Yamaji and G. Saito, Springer-Verlag, Berlin (1998)

${ }^{6}$ Masaki Oshikawa, Phys. Rev. Lett. 84, 3370 (2000)

${ }^{7}$ K. Yamada, K. Yosida and K. Hanzawa, Progress of Th. Phys. 108, 141 (1992)

${ }^{8}$ D. L. Cox and A. Zawadowski, Adv. Phys. 47, 599 (1998) and references therein

${ }^{9}$ A.D. Caplin and J.B. Dunlop, J. Phys. F 3, 1621 (1973)

10 J.B. Dunlop and G. Grüner, Sol. St. Com. 18, 827 (1976)

11 T. Enoki, T. Umeyama, A. Miyazaki, H. Nishikawa, I. Ikemoto and K. Kikuchi Phys. Rev. Lett. 81,3719 (1998)

${ }^{12}$ While in the low temperature insulating phase the magnetic properties are described in terms of the $\mathrm{Fe}^{3+}$ localized spins, in the high temperature metallic phase the CurieWeiss magnetism is described in terms of both $D M E T^{+}$ spins and $\mathrm{Fe}^{3+}$ spins. This behavior is in contradiction to the case of ordinary metals where the conduction electrons give rise to a small Pauli paramagnetic susceptibility with- 
out localized magnetic moments.

${ }^{13}$ G. Nakamoto, T. Takabatake, H. Fujii, A. Minami, K. Maezawa, I. Oguro and A.A. Menovsky, J. Phys. Soc. Japan 64, 4834 (1995)

14 The absence of a gap in these materials can be explained by considering the effect of the Hund's interaction in the virtual $f^{2}$ state, see J. Moreno and P. Coleman, Phys. Rev. Lett. 84, 342 (2000)

${ }^{15}$ H. Ikeda and K. Miyake, J. Phys. Soc. Jpn. 65, 1769 (1996)

${ }^{16}$ F.C. Zhang and T.M. Rice, Phys. Rev. B 37, 3759 (1988)

${ }^{17}$ H. Sato, O.E. Andersson, T. Enoki, I.S. Suzuki and M. Suzuki, J. Phys. Soc. Japan 69. 1136 (2000)

18 T. Schork and P. Fulde, Phys. Rev. B 50, 1345 (1994)

${ }^{19}$ P. Coleman and A.M. Tsvelik, Phys. Rev. B 57, 12757 (1998)

${ }^{20}$ D. L. Cox, Physica B 186-188, 312 (1993)

${ }^{21}$ P. Coleman, A. M. Tsvelik, N. Andrei and H.Y. Kee, Phys. Rev. B 60, 3608 (1999); P. Coleman, A. M. Tsvelik, N. Andrei and H.Y. Kee, J. Phys. Cond. Matt. Letters, 10, L239 (1998)

${ }^{22}$ S. R. White, Phys. Rev. Lett. 69, 2863 (1992), Phys. Rev. B 48, 10345 (1993), Phys. Rep. 301, 187 (1998)

${ }^{23}$ C.C. Yu and S. White, Phys. Rev. Lett. 71, 3866 (1993)

${ }^{24}$ N. Shibata, T. Nishino, K. Ueda and C. Ishii, Phys. Rev.
B 53 R8828 (1996)

${ }^{25}$ M. Guerrero and C.C. Yu, Phys. Rev. B 51, 10301 (1995), M. Guerrero and R.M. Noack, Phys. Rev. B 53, 3707 (1996)

${ }^{26}$ E. S. Sørensen and I. Affleck, Phys. Rev. Lett. 71, 1633 (1993)

27 X. Wang, S. Qin and L. Yu, Phys. Rev. B 60, 14529 (1999)

${ }^{28}$ U. Schollwöck and Th. Jolicoeur, Europhys. Lett. 30, 493 (1995); U. Schollwöck, O. Golinelli and Th. Jolicoeur, Phys. Rev. B 54, 4038 (1996)

${ }^{29}$ N. Shibata, M. Sigrist and E. Heeb, Phys. Rev B 56, 11084 (1997)

${ }^{30}$ We calculate the spin-spin correlation function to be:

$$
\begin{array}{r}
\left\langle\vec{S} \cdot \vec{s}_{i}\right\rangle=3\left\langle S^{z} s_{i}^{z}\right\rangle=3\left\langle S^{z} \frac{\left(\rho_{i \uparrow}-\rho_{i \downarrow}\right)}{2}\right\rangle= \\
=3\left\langle S^{z}\left(\rho_{i \uparrow}-\frac{1}{2}\right)\right\rangle=3\left\langle S^{z} \rho_{i \uparrow}\right\rangle .
\end{array}
$$

The charge-charge correlations function is:

$$
\begin{array}{r}
C(i)=\left\langle\rho_{0} \rho_{i}\right\rangle-\left\langle\rho_{0}\right\rangle\left\langle\rho_{i}\right\rangle=\left\langle\rho_{0} \rho_{i}\right\rangle-1= \\
=2\left[\left\langle\left(\rho_{0 \uparrow}-1 / 2\right)\left(\rho_{i \uparrow}-1 / 2\right)\right\rangle+\left\langle\left(\rho_{0 \uparrow}-1 / 2\right)\left(\rho_{i \downarrow}-1 / 2\right)\right\rangle\right] .
\end{array}
$$

${ }^{31}$ K. Yosida, Phys. Rev. 147, 223 (1966) 